\title{
An alternative to the problem of insufficient physician manpower in the clinical care of sexually transmitted diseases
}

\author{
CHARLES W. PARTON*† AND SUZANNE M. SGROI* \\ From the *Department of Ambulatory and Community Medicine, Mount Sinai Hospital, and the \\ †Department of Community Medicine and Health Care, University of Connecticut Medical School, USA
}

\section{Introduction}

In defining the problem of disproportion in the provision of medical care, it is helpful to divide it into three main categories: geographic, socioeconomic-cultural, and disease-related. The Hartford area in affluent Connecticut, as in other less fortunate areas, has significant distribution problems in each of these three categories. In this paper, however, I shall consider the last of these, the disease-related maldistribution of physician services.

\section{Manpower deficiencies in STD clinics}

Among the new trends in the epidemiology of sexually transmitted diseases it is pertinent to discuss not only the mechanisms and trends of disease processes but also some of the problems that interfere with the management of the disease.

Health departments at all levels in the United States often have difficulty in recruiting physicians with a sustained interest in running well-controlled, high-quality, comprehensive sexually transmitted disease (STD) clinics. Most physicians look on the problem of 'VD' with as much interest and enthusiasm as they do a back-ache.

The reasons for this attitude are many, but two stand out clearly. The health hazards of the various sexually transmitted diseases are not even partially realised by the practising physician and as yet little effort has been spent in our medical schools to change this.

Address for reprints: Dr C. W. Parton, Department of Ambulatory and Community Medicine, Mount Sinai Hospital, 500 Blue Hills Avenue, Hartford, Connecticut 06112, USA

Received for publication 21 September 1978
The belief that syphilis is almost controlled and that its identification is assured by serological testing at premarital examinations and on admission to hospital, coupled with the dramatic response to treatment by penicillin, has rid our streets and our consciousness of the cardiovascular and neurological complications of the syphilis of the past. Furthermore, the ease with which a case of gonorrhoea is treated fails to alert the physician to the significance of the disease. It is our contention, based on several studies, that 65000-100000 young American women become sterile following gonococcal salpingitis each year. Because of the delay and confusion in relating these complications to the primary cause there is little concern among physicians and governments (Sgroi, 1978).

Other examples of inadequate service due to lack of, or suppression of, concern by physicians are: (1) the rising incidence of sexual molestation of children and the resulting STD problems with these children; and (2) the special needs of the male homosexual in whom $50 \%$ of cases of syphilis occur, but for whom society has little understanding.

The second major cause of poor physician distribution is the inadequate pay scale for health department physicians. While the dedicated public health service physician devotes himself or herself to high-quality and conscientious work, the local 'VD clinic physician' is frequently elderly, outmoded, uninterested, or just unobtainable.

\section{Situation in Hartford, Connecticut, in 1975}

The city of Hartford in central Connecticut, halfway between Boston and New York City, has an urban population of 158000 people, about $40 \%$ of whom are black or of Hispanic ethnic origin. The city, whose average annual income is $\$ 13000$ and $38 \%$ of whose population receives city or state welfare benefits, is the capital of one of the wealthiest 
states in the country with its aircraft and light industries, yankee ingenuity, and insurance underwriting. Indeed, Hartford is known as the 'insurance capital of the world'.

The physician-to-population ratio in the city is $1: 614$. There are the University of Connecticut Medical Dental School and three university affiliated community hospitals, and the quality and quantity of medical care is virtually unsurpassed. Nevertheless, in 1974 Hartford's health director was unable to employ a suitable physician to run the city health department's communicable disease clinic.

Since its creation by the Mount Sinai Hospital in 1972, the department of ambulatory and community medicine has advocated a systematic approach to meet.ng community health care needs, especially where there is maldistribution of physicians. It was thus natural that the hospital and the health department entered into a contractual relationship to help to solve this problem.

\section{Hospital and city health department co-operation}

The concept of a community hospital and health department co-operating in a co-ordinated programme is not well established. In negotiations between the City Health Director and the Chief of Community Medicine at the Mount Sinai Hospital the following elements were considered:

(1) The need of the city for physician services.

(2) The ability of the hospital's resources and constraints to meet these needs, based on the fact that (a) the need was real; (b) that there was neither competition with the private practice sector nor duplication of service; (c) the programme was financially feasible; (d) that the hospital could deliver quality care; and (e) that there would be benefits derived to the hospital, such as good public relations, patient referrals, and a challenge for department personnel.

(3) The city's resources included: (a) salaries for the physician, nurse, and clerk; (b) a wellmanaged complete laboratory service; and (c) existing space and equipment.

(4) The city's health department would retain its natural functions of providing services in epidemiology, education, and case-finding.

(5) The state resources supplied most medication and some financing to the city.

(6) The hospital would develop new sources of finance, by charging regular clinic fees to the city and state welfare department, where appropriate. It was noted that in no way must the inability, or even the lack of desire to pay, be a deterrent to full and conscientious management.

The Mount Sinai Hospital department of ambulatory and community medicine, after surveying the needs, available resources, and financial mechanisms, developed the following programme.

\section{PROGRAMME}

\section{Clinical management}

The needs of the clinic demanded accurate diagnostic procedures, including in-depth interviewing covering all aspects of STD and all possible sources and routes of transmission of disease. In addition, adequate general and specific examinations were needed to rule out or to refer elsewhere other health problems which were not managed in the clinic. As regards treatment, the question when, or whether, to treat patients with and without obvious clinical manifestations had to be decided. Finally, appropriate management protocols were needed for each category of STD by age, sex, and special need.

\section{Staff}

In approaching the problem of maldistribution of physicians our department has developed pools of nurse practitioners, physician associates, and physicians, who rotate through various assignments, no one of which would necessarily be stimulating on a permanent basis but which together provide a varied and exciting medical challenge (Figure).

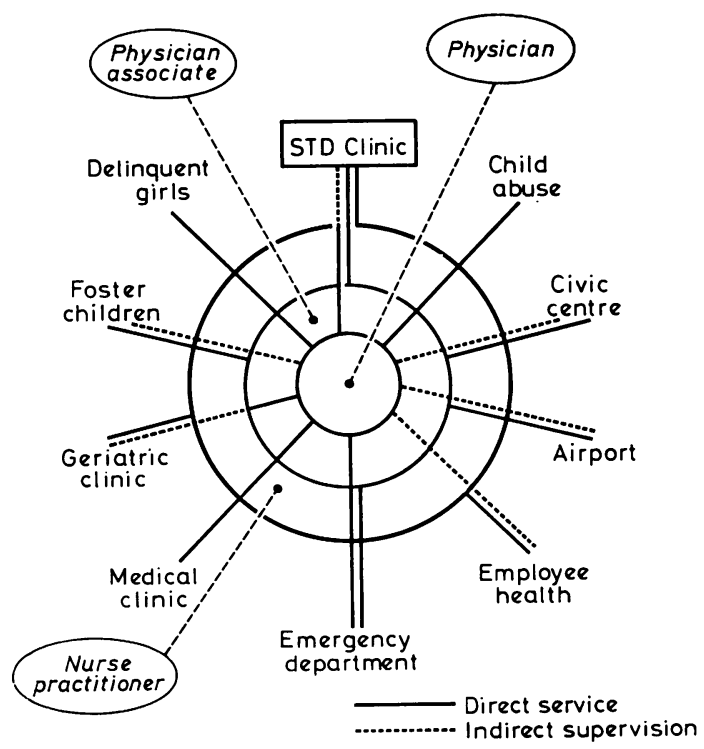

Figure Mount Sinai Hospital outreach programme 
We believed that the physician associates, who are all college graduates with two years of accredited study as generalists and who are all certified by national board examinations, could provide a high level of skill at considerably lower cost; the availability of personnel was assured through rotation. There would also be a need for nurses in the clinic, but their role could also be expanded to provide equally capable diagnostic and treatment skills. In providing this service, however, we were responsible for the quality, reproducibility, and continuity of care. We planned, therefore, a specialised training in STD for these two groups.

\section{Training in STD}

Dr Suzanne Sgroi, our clinic director, having first developed a management protocol, undertook the design and production of a training course, which consisted of 15 hours of didactic lectures and reading assignments followed by 15 hours of directly supervised training in the clinic. This latter section, which follows successful completion of a written examination, provides preceptorial clinic experience followed by practical experience under direct supervision, so that the student not only sees how it is done but does it with continuous critique.

The hospital's clerical personnel, who have also participated in the educational programme, provide invaluable help in answering the telephone. They convey to callers our desire to help by their warmth and understanding.

\section{Finance}

The city health department has had no increase in costs over their pre-contract budget. There are now more employees providing more service to more patients than ever before. Clearly one source of savings has been the use of physician assistants and nurse practitioners in the clinic instead of physicians. The hospital now bills city and state insurance schemes, and is investigating direct collections for those patients who volunteer to pay for their service-which to our surprise was common. We make it abundantly clear that no patient will be denied treatment for lack of ability, or indeed lack of desire, to pay. So far we have had no complaint or loss of clientele.

\section{Physician supervision}

We were also concerned with physician supervision and audit of the quality of care. This component is provided by our physicians under the clinic director. These physicians attend in rotation for three hours each day and provide supervision in the clinic. They directly observe physician associates and nurse practitioners, and are consulted on specific problems; they carefully review each patient's case record for completeness and compliance with our protocol.

During the clinic hours when a physician is not present patients are diagnosed and treated by the physician assistant and nurse practitioner groups following the protocol. There is indirect physician supervision by telephone from a nearby primary care practice clinic or from the hospital emergency department.

In the past year one full-term equivalent (FTE) nurse practitioner and one FTE physician assistant from our pool of eight physician assistants, who rotate services, cared for 9000 patients at a unit treatment cost of less than $\$ 10 \cdot 00$.

\section{Conclusions}

Our programme demonstrates that high-quality clinical management of STDs, including diagnosis, treatment, and education, can be delivered by physician extenders working under direct or indirect physician supervision and using a protocol. The programme demands a thorough education in disease processes, epidemiology, and treatment under close medical scrutiny.

We believe this care to be more thorough and delivered with greater enthusiasm, compassion, and interest than is available in some clinics in the USA, in which all the clinical care is provided by physicians.

\section{Reference}

Sgroi, S. M. (1978). Venereal disease: hazardous to a woman's health, VD News, vol. 3, No. 1 . 